\title{
EXOPLANETS
}

\section{Know thy star, know thy planet - or not?}

Astron. J. 158, 239 (2019)

Exoplanet formation models usually rely on the assumption that the more metals in the host star, the more metals in the giant planets of the system - since more metals means more solids in the feeding zone of the forming planet in its protoplanetary disk. However, Johanna Teske and collaborators have conducted a detailed study of 22 exoplanet systems and show that this is not the case: there is no metallicity 'enhancement' for giant planets in metal-rich systems; the metal content of a giant planet depends upon its mass.

The authors calculate abundances of $\mathrm{Fe}$, $\mathrm{Mg}, \mathrm{Si}, \mathrm{Ni}, \mathrm{O}$ and $\mathrm{C}$ in the systems' host stars, following a dedicated spectroscopic survey and subsequent Bayesian analysis, and calculate metallicities for their cool $(<1,000 \mathrm{~K})$ giant planets by employing a one-dimensional hydrostatic equilibrium model using radius and mass measurements. A linear relationship between stellar and planetary metal abundances (aggregated) can be excluded at the $2 \sigma$ level. There is no clear explanation for this lack of a trend, but the authors suggest that a disk process that regulates solid surface densities (for example, radial drift of grains) could be at work, or perhaps that the orbit at which a planet forms in the disk interferes with the observed relationship.

The authors also uncover a tentative correlation between residual metal abundance (the relative amount of metals compared to that expected due to planet mass alone) and the ratio of volatile element $(\mathrm{C}, \mathrm{O})$ enrichment to refractory element enrichment: giant planets with more metals than expected have stars with a higher degree of volatile elements.

\section{Paul Woods}

Published online: 6 January 2020

https://doi.org/10.1038/s41550-019-1005-7 